Supporting information for:

\title{
Virus-enabled optimization and delivery of the genetic machinery for efficient unnatural amino acid mutagenesis in mammalian cells and tissues
}

Yunan Zheng, ${ }^{1}$ Tommy Lewis $\mathrm{Jr}^{2},{ }^{2}$ Peter Igo, ${ }^{1}$ Franck Polleux,${ }^{2}$ and Abhishek Chatterjee ${ }^{1} *$

${ }^{1}$ *Department of Chemistry, Boston College, 2609 Beacon Street, Chestnut Hill, MA 02467, USA

${ }^{2}$ Department of Neuroscience, Columbia University, 550 West 120th Street New York, NY 10027, USA

*email: abhishek.chatterjee@bc.edu 


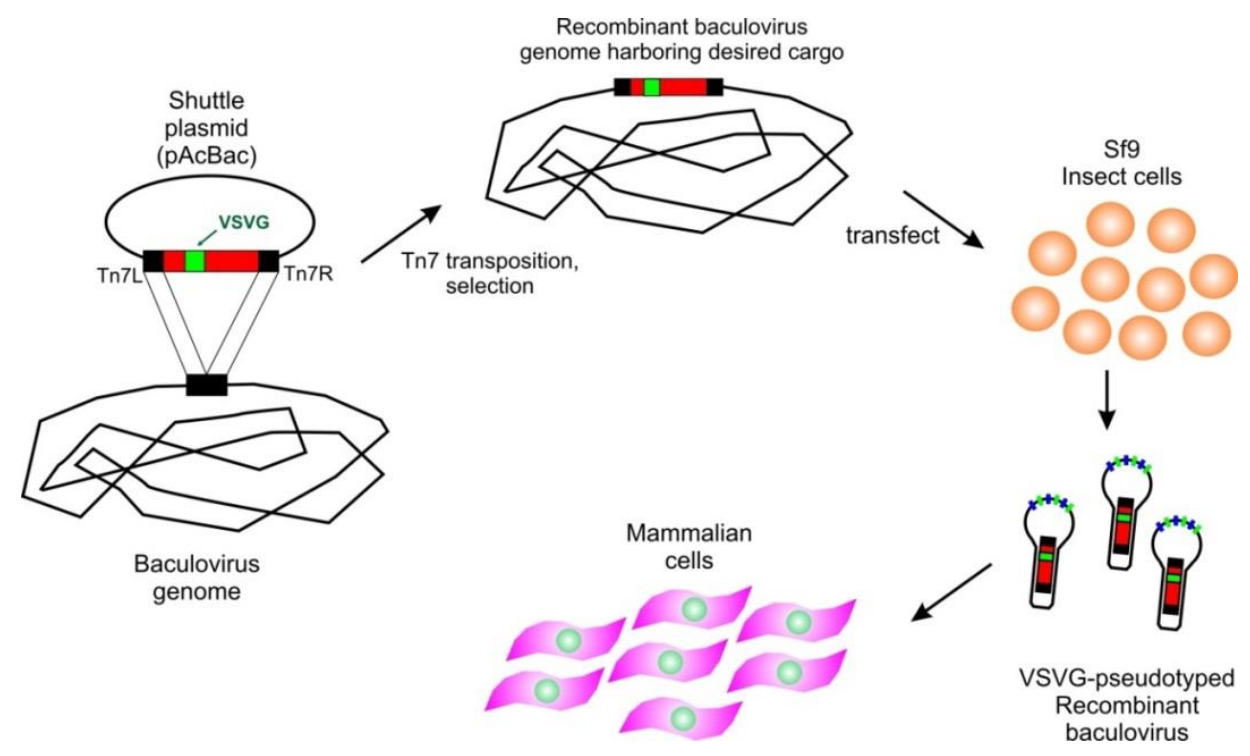

Figure S1: Generating recombinant, VSVG-pseudotyped baculovirus to deliver genetic cargo into mammalian cells. 


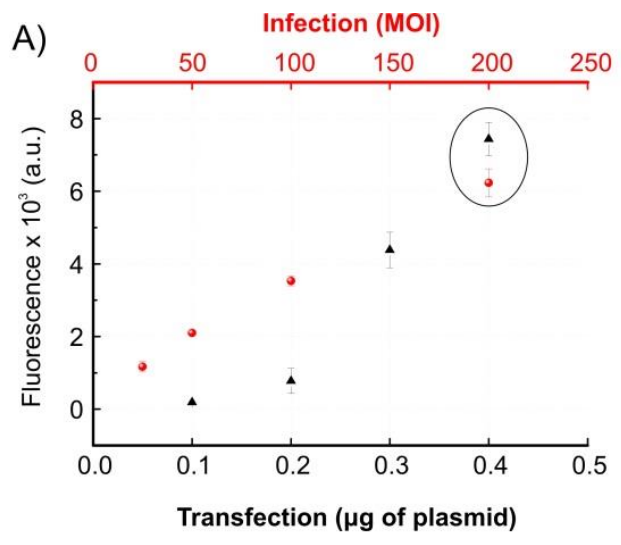

B)
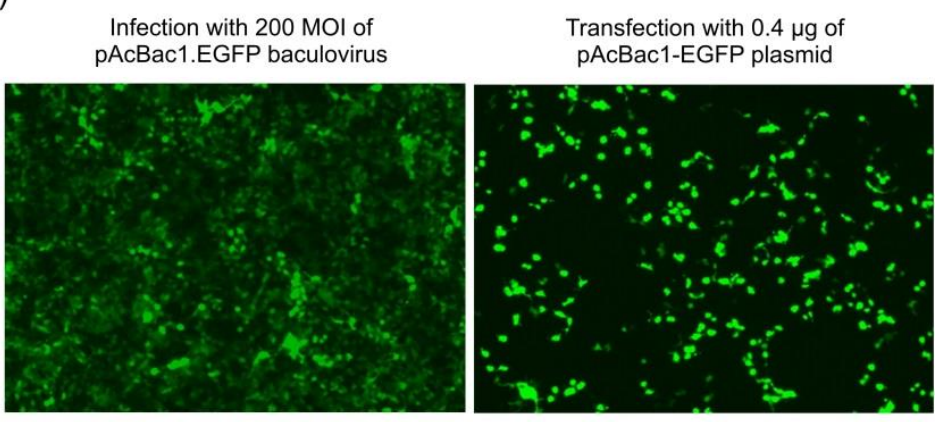

C)
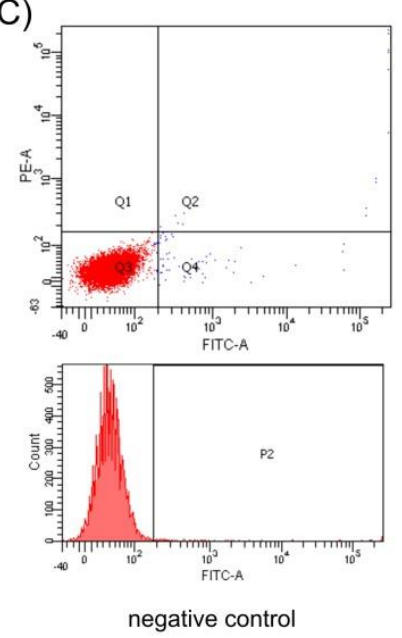

D)
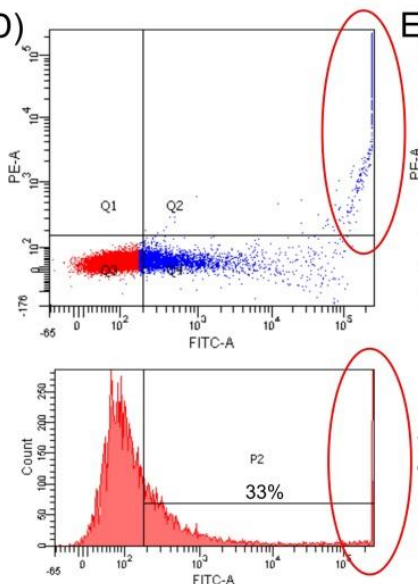

Transfection with $0.4 \mu \mathrm{g}$ of
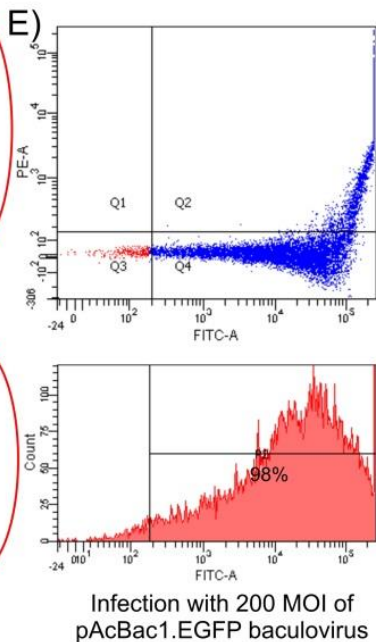

F)
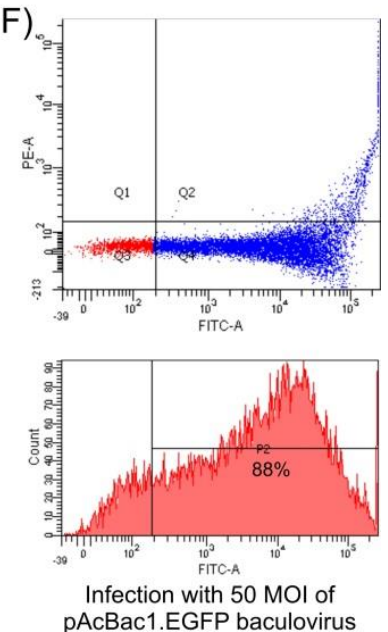

Figure S2: Comparing the expression patterns resulting from the delivery of pAcBac1.EGFP (Figure S3) into HEK293T cells by two methods, transient transfection and baculovirus mediated infection. A) Expression of EGFP (wild-type) in HEK293T cells (measured as fluorescence in clarified cell-lysate) in response to increasing amounts of transfected DNA (black triangles) or increasing MOI of the baculovirus encoding the same cargo (red circles). B) Fluorescence images showing the expression pattern of EGFP in transfected or infected HEK293T cells (confluent) that produced comparable amounts of total EGFP (indicated by the black ellipse in panel A). FACS analysis of untreated HEK293T cells (C), cells transfected with $0.4 \mu \mathrm{g}$ of pAcBac1.EGFP plasmid (D), cells infected with 200 MOI of pAcBac1.EGFP baculovirus (E), and cells infected with $50 \mathrm{MOI}$ of pAcBac1.EGFP baculovirus (F). Fluorescent cells with very high EGFP expression could not be displayed within the dynamic range of this setting (indicated with the red ellipse in D), but are included in the calculation of the fluorescent population. 

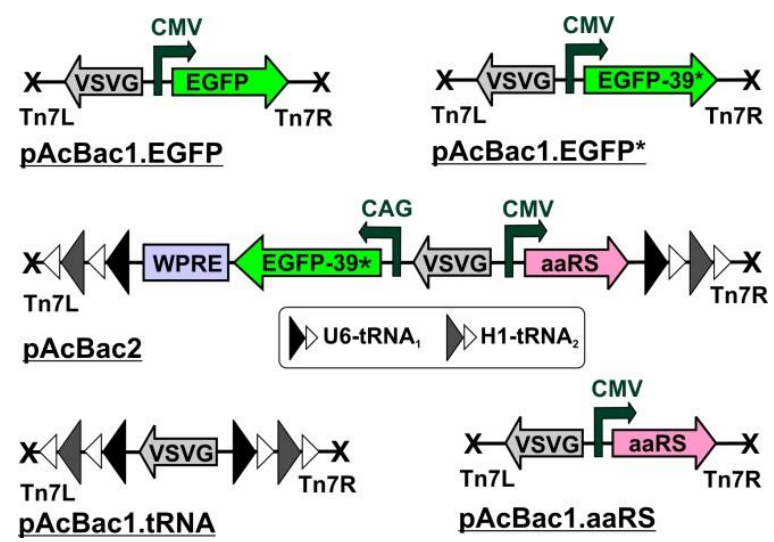

Figure S3: Maps of various baculovirus shuttle vectors used in this study. 

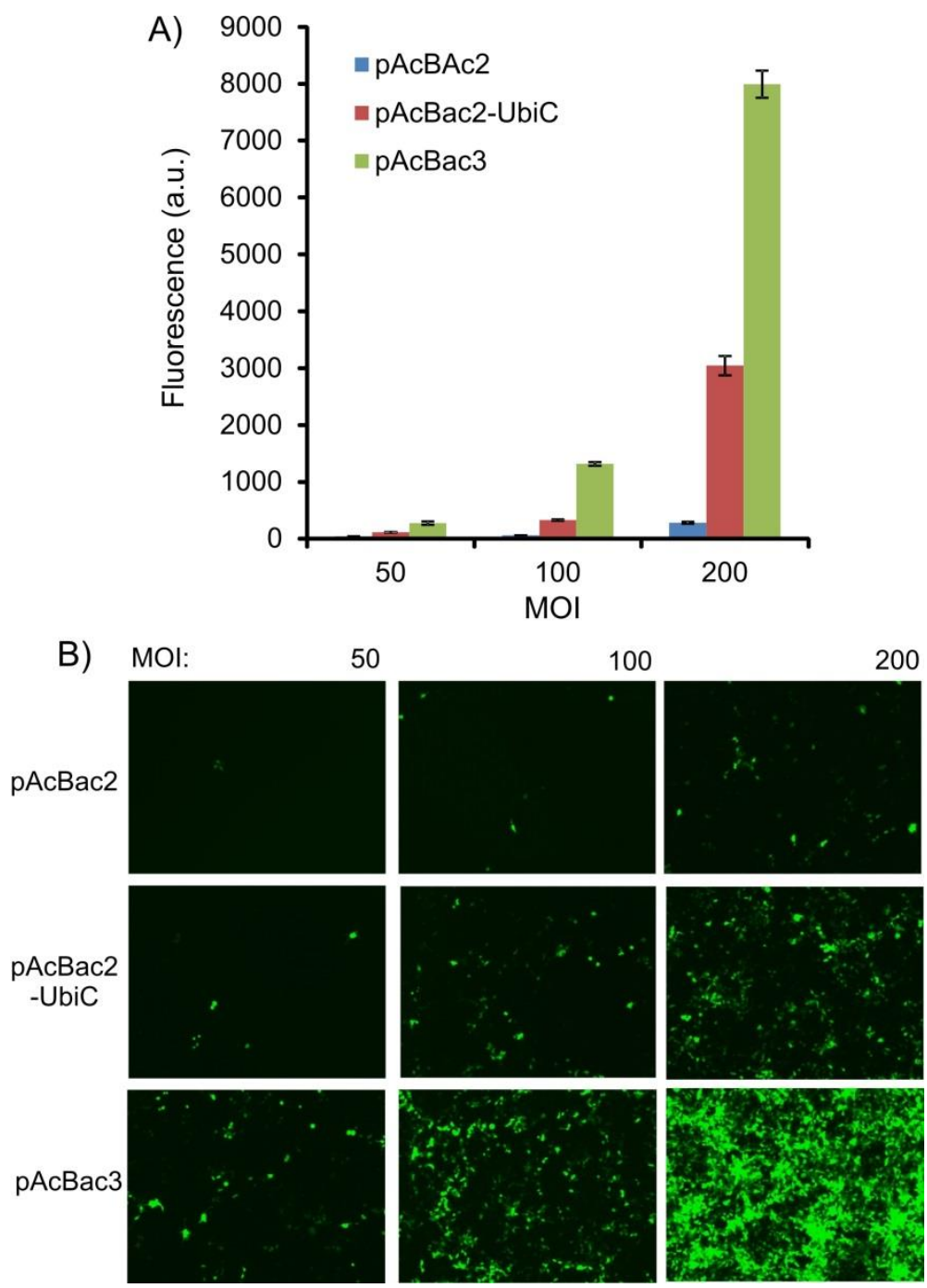

Figure S4: EGFP* expression in HEK293T cells infected with pAcBac2, pAcBac2-UbiC, and pAcBac3 at various MOI shown as: A) EGFP-fluorescence in cell-lysate; or B) Fluorescence images of a confluent monolayer of cells. Analysis was performed $48 \mathrm{hr}$ post-infection and $1 \mathrm{mM} \mathrm{OMeY}$ was incorporated in the culture medium. 

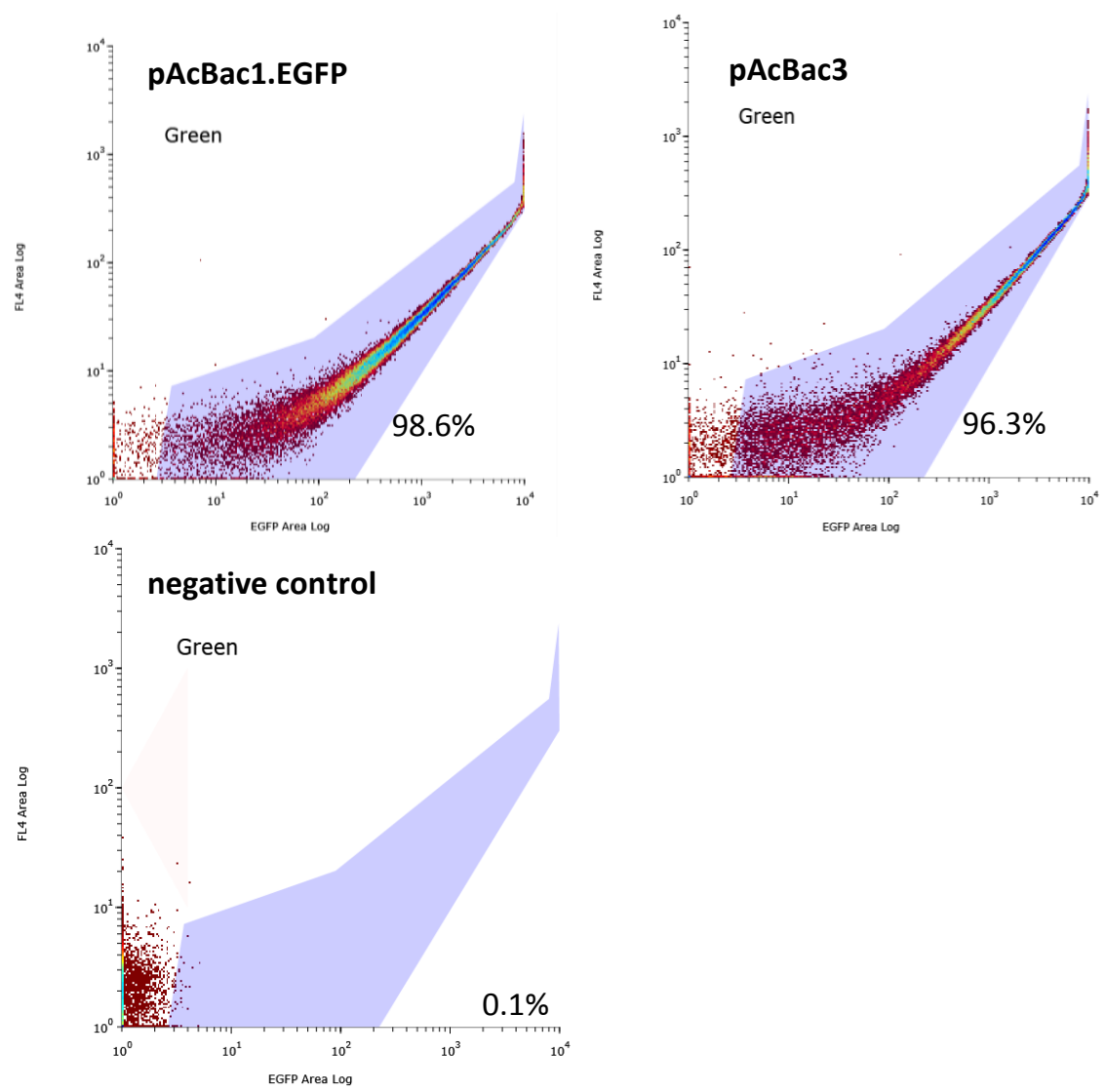

Figure S5: FACS analysis of HEK293T cells infected with 200 MOI of pAcBac1.EGFP, pAcBac3 (in the presence of $1 \mathrm{mM} \mathrm{OMeY}$ ), or no virus (negative control). \% of population expressing EGFP (cells residing within the shown gate are also mentioned. 


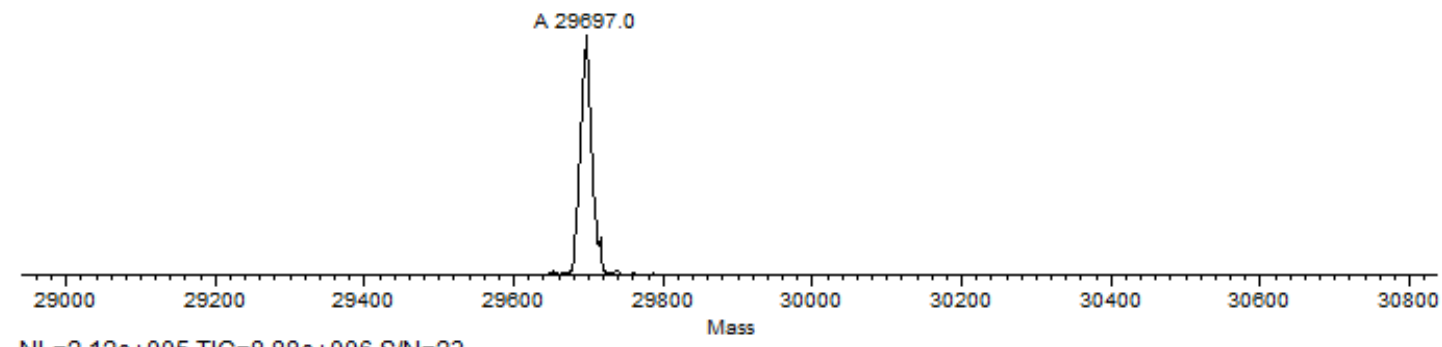

$\mathrm{NL}=2.12 \mathrm{e}+005 \mathrm{TIC}=8.88 \mathrm{e}+006 \mathrm{~S} / \mathrm{N}=23$

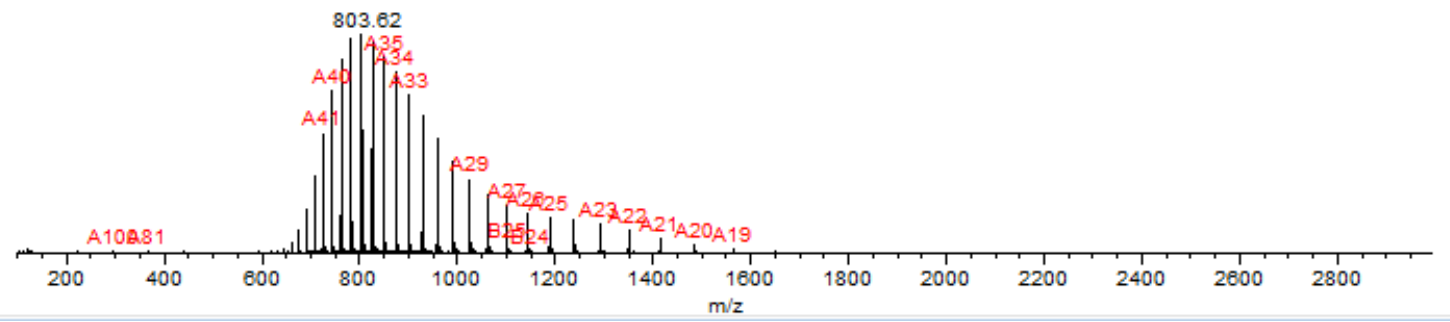

Figure S6: MS analysis of EGFP-39-OMeY isolated from HEK293T cells using Ni-NTA affinity chrmoatography show a homogeneous species with the expected mass (expected: 29698 Da, observed $29697 \mathrm{Da}$ ). Bottom panel shows the $\mathrm{m} / \mathrm{z}$ peak enevelop for the protein, top panel shows the deconvoluted mass. 


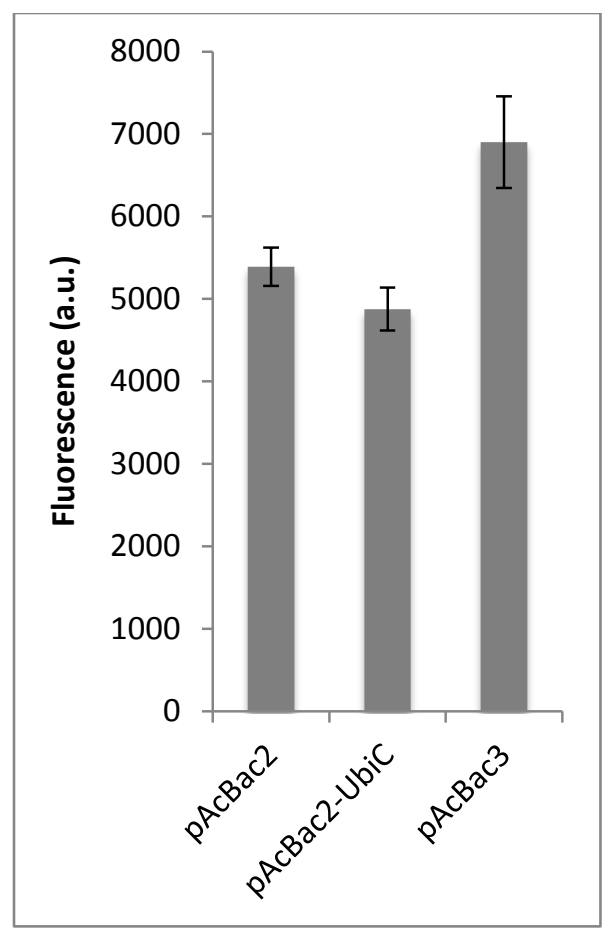

Figure S7: Expression level of EGFP* upon transfecting HEK293T cells with plasmids harboring pAcBac2, pAcBac2-UbiC, and pAcBac3 expression systems, measured as EGFP-fluorescenece in celllysate. $1 \mathrm{mM} \mathrm{OMeY}$ was used as the UAA in the medium, and the analysis was performed $48 \mathrm{hr}$ posttransfection. Comparable expression levels of the three systems demonstrate the obscuring of their intrinsic differences due to the overexpression of the encoded machinery in transiently transfected cells. 


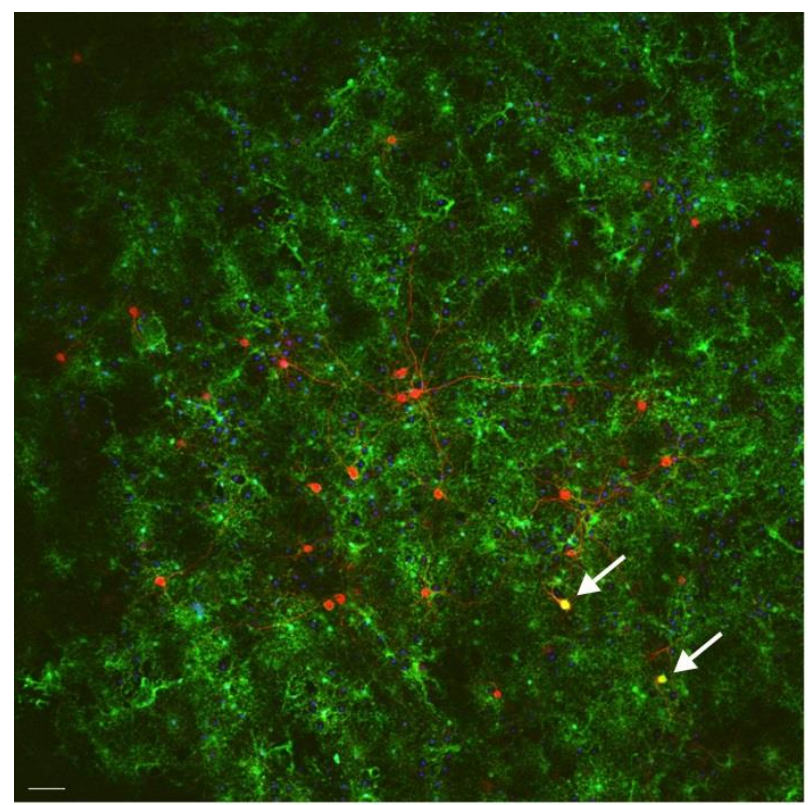

$+1 \mathrm{mM} \mathrm{pAcF}$

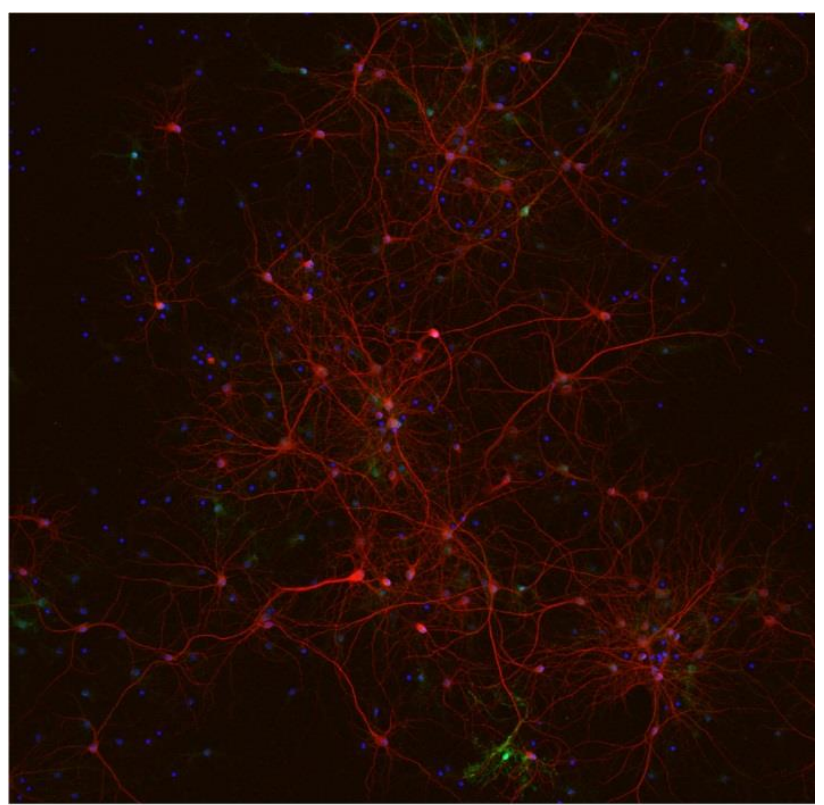

$-1 \mathrm{mM}$ pAcF

Figure S8: Expression of EGFP* in the dissociated primary mouse brain-tissue monolayer culture composed of neurons and glial cells. EGFP fluorescence is pseudo-colored in green, DAPI (nuclear stain) is pseudo-colored in blue, and neuron-specific MAP2 marker is pseudo-colored in red. $<5 \%$ of neurons in the co-culture expressed EGFP (indicated by arrow), while the majority of reporter expression was associated with glial cells. 

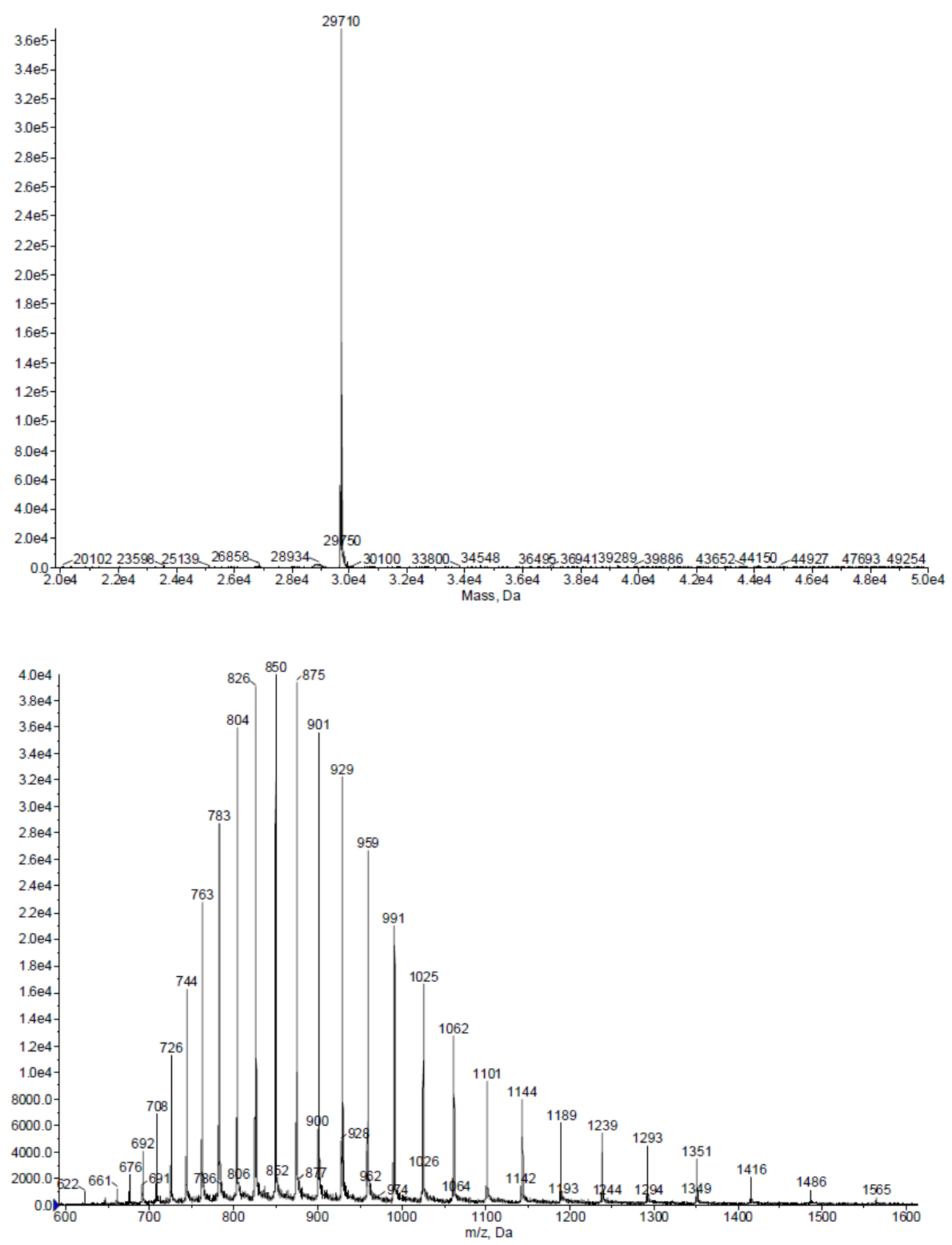

Figure S9: MS analysis of EGFP-39-pAcF isolated from a monolayer co-culture of mouse brain tissue using Ni-NTA affinity chrmoatography show a homogeneous species with the expected mass (29710 Da). Bottom panel shows the $\mathrm{m} / \mathrm{z}$ peak enevelop for the protein, top panel shows the deconvoluted mass. 


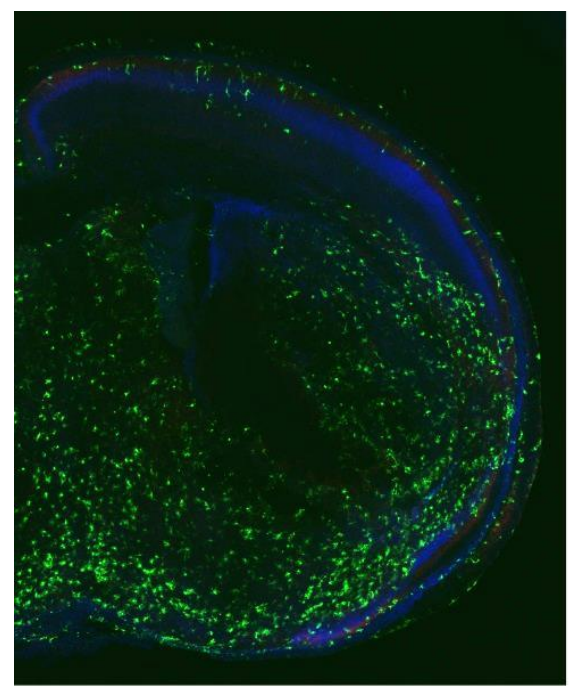

$+1 \mathrm{mM}$ pAcF

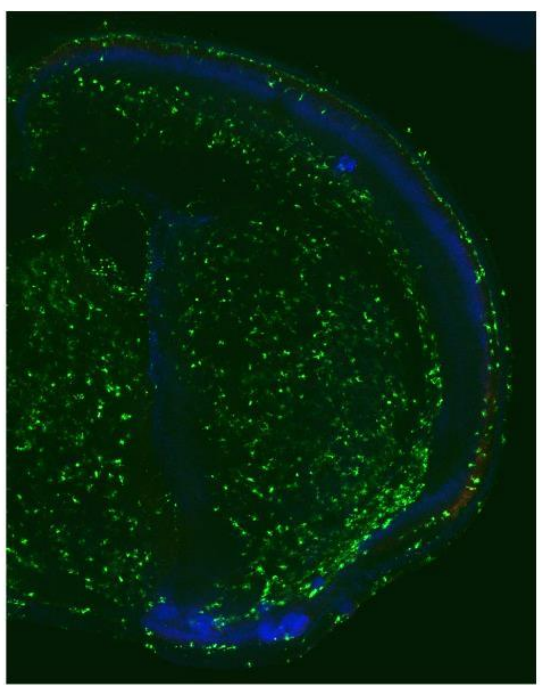

$+1 \mathrm{mM} p A z F$

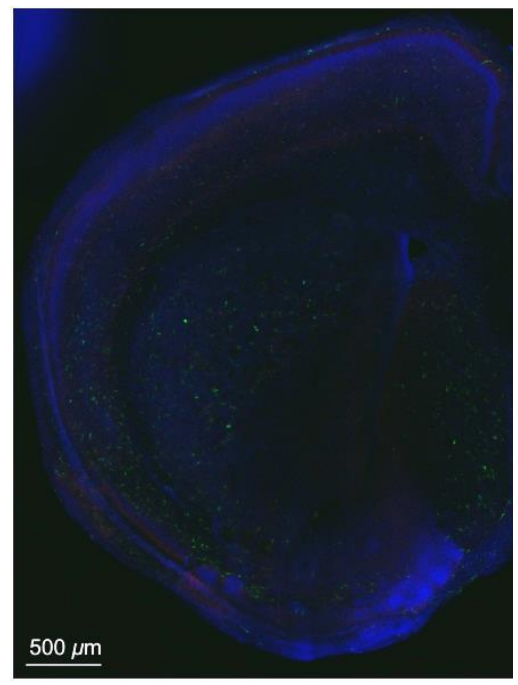

no UAA

Figure S10: Fluorescence images of EGFP* expression in mouse brain slices overlaid with $10^{8}$ infective units of pAcBac3, in the presence of $1 \mathrm{mM} \mathrm{pAcF}$ or pAzF, or in the absence of any UAA. Co-stained with DAPI (blue). 


\section{Materials and methods:}

\section{General}

DH10B (Life Technologies, Carlsbad, CA) strain of E. coli was used for cloning and plasmid propagation. Bacteria were grown on LB/agar plates and LB liquid medium with the following antibiotic concentrations unless otherwise mentioned: $100 \mu \mathrm{g} / \mathrm{mL}$ ampicillin, $10 \mu \mathrm{g} / \mathrm{mL}$ gentamycin, $50 \mu \mathrm{g} / \mathrm{mL}$ kanamycin, $10 \mu \mathrm{g} / \mathrm{mL}$ tetracycline. Phusion Hot Start II DNA polymerase (Fisher Scientific, Waltham, MA) was used for PCR reactions using protocol provided by the manufacturer. Restriction enzymes and T4 DNA ligase were obtained from NEB (Ipswich, MA). For DNA purification (plasmid as well as PCR products, etc.), spin columns from Epoch Life Science (Fort Bend County, TX) were used. DNA oligomers synthesis and Sanger DNA sequencing was performed by IDT (Coralville, IA) and Eton Biosciences (Charlestown, MA), respectively. Various antibiotics were purchased from Fisher Scientific (Waltham, MA). For baculovirus expression, DH10Bac E. coli cells and Sf9 cells are obtained from Life Technologies (Carlsbad, CA), and Sf-900 III SFM media for insect cell culture was purchased from Fisher Scientific (Waltham, MA). BacPAK Baculovirus Rapid Titer Kit Clontech (Mountain View, CA) was used according to the manufacturer's protocol to evaluate the titer of the baculovirus preparations. For mammalian cell culture, DMEM/high glucose media and trypsin $0.25 \%$ (1X) solution were obtained from GE Healthcare Life Sciences (Logan, UT). Fetal Bovin Serum (FBS) is obtained from Corning (Manassas, VA).

Construction of viral vectors The previously reported $\mathrm{pAcBac} 1$ and $\mathrm{pAcBac} 2$ vectors were used to generate additional plasmids. ${ }^{1}$ To build the pAcBac1 plasmids that only express the aaRS (pAcBac1.aaRS) or the EGFP-39TAG (pAcBac1.EGFP*), the DNA sequence encoding the EcTyrRS synthetase or the EGFP-Tyr39TAG - both driven by a CMV-IE promoter - were obtained by digesting pAcBac1-tR4-OMeYRS or pAcBac1-tR4-GFP*, ${ }^{1}$ respectively, with NotI/EcoRI, and inserting into digested pAcBac1 vector cut with the same pair of restriction enzymes. The previously described vector pAcBac1.tR4 was used as pAcBac1.tRNA. ${ }^{1}$

To generate pAcBac2 vectors with alternative promoters driving aaRS expression, UbiC, hPGK and SV40 promoter sequences were amplified (sequence given below) using primers listed below. Corresponding PCR products were ligated into pAcBac2 following digestion with SpeI/SacI, such that the new promoter replaces the preexisting CMV-IE promoter.

In order to construct a viral vector that contains 16 tandem copies of the tRNA expression cassette, first a two-copy tRNA cassette encoding a Bacillus stearothermophilus (Bst) tRNA ${ }_{\mathrm{CUA}}{ }^{\text {Tyr }}$ driven by $\mathrm{H} 1$ and an E. coli $\mathrm{tRNA}_{\mathrm{CUA}}{ }^{\mathrm{Tyr}}$ driven by U6 promoter $(2 \mathrm{xtRY})$ was PCR amplified from pAcBac1-tR4 ${ }^{1}$ and inserted into pIDT-Kan (IDT, Coralville, IA) vector between NheI and AvrII to generate pIDT-Kan-2xtRY. This vector was linearized by digesting with AvrII alone, and an additional copy of the 2xtRY (generated by digesting pIDT-Kan-2xtRY with AvrII/NheI) was cloned into it. Since AvrII and NheI sites contain compatible ends, ligation of the new 2xtRY retains the AvrII site only on one of the termini (Scheme S1). The clones where the incoming $2 x t R Y$ is aligned in the same direction with the preexisting $2 x t R Y$ cassette were identified by AvrII/NheI digestion to obtain a pIDT-Kan-4xtRY. Repeating this procedure again on pIDT-Kan-4xtRY produced pIDT-Kan-8xtRY, and next pIDT-Kan-16xtRY (Scheme S1). Finally, the 16xtRY was released from pIDT-Kan-16xtRY by AvrII/NheI digestion and was cloned into a compatible SpeI site in pAcBac2-UbiC to generate pAcBac3 (map and sequence below). 


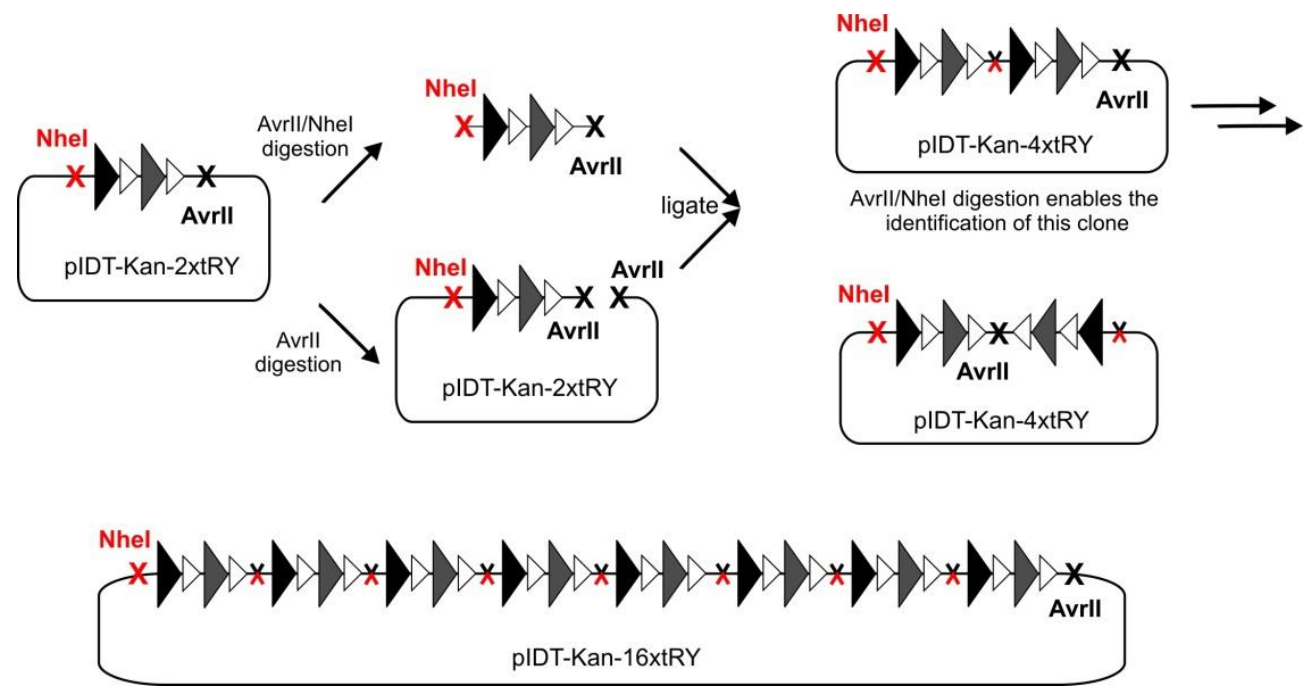

Scheme S1: Generation of 16x-tandem tRNA expression cassettes.

To generate pAcBac2 and pAcBac2-UbiC with a C-terminally Myc-tagged OMeYRS, DNA sequence of OMeYRS was PCR amplified using a reverse primer (EcYRS-Myc-EcoRI-R) that introduced the Myc sequence, and was cloned between EcoRI and NheI sites in pAcBac2 and pAcBac2-UbiC, replacing the pre-existing OMeYR.

\section{List of Primers}

SV40-Spe-F: AATAATACTAGTGGCCTATTGGTTAAAAAATGAGCTGATTTAACAAAAATTTAACGCG SV40-Sac-R: AATAATGAGCTCCCGGGAGCTTTTTGCAAAAGCCTAGGC hPGK-Spe-F: AATAATACTAGTGGGTTGCGCCTTTTCCAAGGCAGCCCTGG hPGK-Sac-R: AATAATGAGCTCCTGGGGAGAGAGGTCGGTGATTCGGTCAACG UbiC-Spe-F: AATAATACTAGTGGCCGTTCGAAGGCCTCCGCGCCG UbiC-Sac-R: AATAATGAGCTCTAGACCAAGTGACGATCACAGCGATCCACAAAC tR-Avr-F: AATAATCCTAGGTCGGGCAGGAAGAGGGC tR2-Nhe-R: CGTCGATCCGCTAGCAAAAAATGGAGGGG EcYRS-Myc-EcoRI-R: AATAATGAATTCTTACAGATCCTCTTCAGAGATGAGTTTCTGCTCCCCAACGGGCCCTTTCCAGCAAAT CAG EcYRS-NheI-F: AATAATGCTAGCGCCACCATGGCAAGCAGTAAC

Sequence of SV40, hPGK and UbiC promoters:

\section{UbiC promoter:}

ACTAGTggccgttcgaaggcctccgcgccgggttttggcgcctcccgcgggegccccctcctcacggcgagcgctgccacgtcagacgaaggg cgcagcgagcgtcctgatccttccgcceggacgctcaggacagcggccegctgctcataagactcggccttagaaccccagtatcagcagaaggacatttaggacgg gacttgggtgactctagggcactggttttctttccagagagcggaacaggcgaggaaaagtagtccettctcggcgattctgcggagggatctccgtggggcggtgaac gccgatgattatataaggacgcgecgggtgtggcacagctagttccgtcgcagccgggatttgggtcgcggttcttgtttgtggatcgctgtgatcgtcacttggtctaGA $\underline{\text { GCTC }}$

\section{hPGK promoter:}

ACTAGTgggttgcgccttttccaaggcagccctgggtttgcgcagggacgcggctgctctgggcgtggttccgggaaacgcagcggegccgaccct gggtctcgcacattcttcacgtccgttcgcagcgtcacccggatcttcgecgctaccettgtgggecccceggcgacgettcctgctcegcccctaagtcgggaaggttcc ttgcggttcgcggegtgccggacgtgacaaacggaagccgcacgtctcactagtaccctcgcagacggacagcgccagggagcaatggcagcgegccgaccgcga 
tgggctgtggccaatagcggctgctcagcagggcgcgccgagagcagcggccgggaaggggcggtgcgggaggcggggtgtggggcggtagtgtgggccetgtt cetgccegcgeggtgttccgcattctgcaagectccggagcgcacgtcggcagtcggctccetcgttgaccgaatcaccgacetctetccccaGAGCTC

\section{SV40 promoter:}

ACTAGTggcctattggttaaaaaatgagctgatttaacaaaaatttaacgcgaattaattctgtggaatgtgtgtcagttagggtgtggaaagtccccagg ctccccagcaggcagaagtatgcaaagcatgcatctcaattagtcagcaaccaggtgtggaaagtccccaggctccccagcaggcagaagtatgcaaagcatgcatct caattagtcagcaaccatagtccegeccetaactccgcceatccegccectaactccgcccagttcegcccattctcegccecatggctgactaatttttttatttatgcaga ggccgaggccgcctctgcctctgagctattccagaagtagtgaggaggctttttggaggcctaggcttttgcaaaaagctcccggGAGCTC

Insect cell culture and the preparation of Recombinant Baculovirus Recombinant baculovirus was produced as described previously (Figure S1). ${ }^{1}$ Briefly, the pAcBac shuttle plasmids were transformed into DH10Bac (Invitrogen) to transfer the genetic cargo into the baculovirus genome maintained as a bacmid in this cell via Tn7 transposition. Successful transposition events were identified by a blue/white lacZ selection, and the recombinant bacmid was purified from the white colonies. Sf9 cells were cultured in Sf-900 III SFM serum-free media (Fisher Scientific, Waltham, MA) in shaking flask in a refrigerated incubator maintained at $28^{\circ} \mathrm{C}$. Primary baculovirus stock was generated by transfecting adhered Sf9 cells in a T25 flask with the recombinant bacmid (4 $\mu \mathrm{g})$. After 4 days of incubation, the virus-containing supernatant was harvested 96 hours post-transfection and was clarified by centrifugation. The primary virus stock was amplified in a $40 \mathrm{~mL}$ Sf9 suspension culture. The secondary virus was harvested 72 hours post infection using the following procedures: 1) centrifuge the $40 \mathrm{ml}$ culture at $200 \mathrm{xg}$ for 20 minutes to pellet the Sf9 cells; 2) transfer the supernatant containing virus into JA-17 tubes and centrifuge at 39000 $\mathrm{xg}$ for $1 \mathrm{hr}$ to pellet the baculovirus; 3) resuspend the virus pellet in $300 \mu \mathrm{L} \mathrm{PBS}+10 \% \mathrm{DMSO}$; $)$ aliquot the virus stock, flash-freeze them in liquid nitrogen and store in $-80^{\circ} \mathrm{C}$ freezer. The BacPAK Baculovirus Rapid Titer Kit (Clontech, Mountain View, CA) was used to determine infective titers of the virus stocks following manufacture's protocol.

Mammalian cell culture, expression analysis using EGFP fluorescence HEK293T cells were cultured in Dulbecco's modified Eagle's medium (high glucose DMEM) supplemented with $10 \%$ fetal bovine serum (FBS) and Penicillin/Streptomycin $(0.5 \mathrm{X})$ and incubate in $37{ }^{\circ} \mathrm{C}$ with $5 \% \mathrm{CO}_{2}$. Cells were seeded at a density of 200,000 per well for a 12-well plate the day before infection or transfection. For transfection, polyethylenimine (PEI) and DNA was mixed at a ratio of $4 \mu \mathrm{L}$ PEI $(1 \mathrm{mg} / \mathrm{mL})$ to $1 \mu \mathrm{g}$ DNA in DMEM (21 $\mu \mathrm{L}$ per $1 \mu \mathrm{g}$ of DNA), and incubated for $20 \mathrm{~min}$. To investigate the properties of transfection-mediated delivery, various amounts of this transfection mixture was added to the cells. UAAs were added to the culture medium to a final concentration of $1 \mathrm{mM}$ at the same time as transfection/ infection. Fluorescence images and EGFP-expression analysis were performed 48 hours post infection or transfection. To obtain EGFP expression data, cells were harvested and washed once with PBS buffer, and then lysed with CelLytic-M lysis Buffer (Sigma; supplemented with 1X Halt protease inhibitor and $0.01 \%$ Pierce universal nuclease). For a single well of a 12 -well plate, $50 \mu \mathrm{L}$ lysis buffer was used, and after $20 \mathrm{~min}$ incubation the lysate was clarified by centrifuging at $8000 \mathrm{rpm}$ for 5 minutes, and was transferred into a clear bottom 96-well assay plate (Corning, Corning, NY). EGFP fluorescence in lysate was recorded using a SpectraMAX M5 (Molecular Devices, Sunnyvale, CA) using $480 \mathrm{~nm}$ excitation and $530 \mathrm{~nm}$ emission. Isolation of mutant EGFP and its MS analysis was performed as described before. ${ }^{1}$

Western blot analysis HEK293T cells were harvested and lysed as described above $48 \mathrm{hr}$ post-infection, and the clarified lysate was resolved using 12\% SDS-PAGE. Resolved proteins were transferred from the gel to a PVDF membrane (Life Technologies, Carlsbad, CA) using a Trans-Blot Turbo Transfer System 
(Bio-Rad, Heacules, CA). After transferring, the membrane was blocked with blocking solution (5\% nonfat milk and $0.1 \%$ Tween 20 (Fisher Scientific, Waltham, MA) in Tris-buffered saline (TBS) overnight at $4{ }^{\circ} \mathrm{C}$. Next, the blocking solution was removed and the primary antibody (please see below for the antibodies and the dilution used) was incubated with membrane for 1 hour with gentle shaking at RT. The solution with the primary antibody was then removed, and the membrane was washed 6-8 times (10 min incubation with shaking in between) wash solution $(0.1 \%$ Tween 20 in TBS). Then the secondary antibody was added at the appropriate dilution, and was allowed to incubate at RT for 1 hour with shaking, and the wash step was repeated again. Finally, the membrane was developed using SuperSignal West Dura Kit (Fisher Scientific, Waltham, MA) and incubated for 5 minutes before imaging using a ChemiDoc MP imaging system (BioRad). Antibodies and concentrations were as follows: mouse anti- $\beta$ actin loading control antibody (BA3R, Fisher Scientific, Waltham, MA) used at 1:5000 dilution; mouse anti-Myc epitope tag antibody (Myc.A7, Thermo Scientific, Waltham, MA) used at 1:2000 dilution; Living Colors mouse anti-EGFP antibody (JL-8, Clontech, Mountain View, CA) used at 1:2000 dilution, chicken anti-mouse IgG secondary antibody-HRP conjugate (Fisher Scientific, Waltham, MA) used at 1:3000 dilution.

FACS Analysis HEK293T cells were subjected to FACS analysis 48 hours post infection/transfection using the following the procedure: 1) remove the media and add $200 \mu \mathrm{L}$ of trypsin $0.25 \%$ (1X) solution (GE Healthcare Life Sciences, Logan, UT) to each well of a 12-well plate and incubate at $37{ }^{\circ} \mathrm{C}$ for 2 minutes to detach the cells from the plate; 2) add $400 \mu \mathrm{L}$ of DMEM $+10 \%$ FBS to quench the trypsin and transfer the $600 \mu \mathrm{L}$ cell suspension gently into a microcentriguge tube and centrifuge at $200 \mathrm{xg}$ for 3 minutes to pellet the cells; 3) discard the supernatant and wash the cell pellet gently with PBS twice; 4) resuspend the cell pellet to a final density of 2 million cells $/ \mathrm{ml}$ and keep on ice till analysis. FACS analysis was performed using a Bio-Rad S3e cell sorter (Bio-Rad, Hercules, CA) or a BD FACSAria cell sorter.

Animals All animals were handled according to protocols approved by the Institutional Animal Care and Use Committee (IACUC) at Columbia University. Time-pregnant CD1 females were purchased from Charles Rivers while B6 mice were bred in house.

Primary cortical culture Cortices from E15.5 B6 mouse embryos were dissected in Hank's Buffered Salt Solution (HBSS, Life Technologies) supplemented with HEPES (2.5 mM), $\mathrm{CaCl}_{2}(1 \mathrm{mM}$, Sigma), $\mathrm{MgSO}_{4}$ (1 mM, Sigma), $\mathrm{NaHCO}_{3}(4 \mathrm{mM}$, Sigma) and D-glucose (30 mM, Sigma), hereafter referred to as cHBSS. Cortices were dissociated in cHBSS containing papain (Worthington) and DNAse I (100 $\mu \mathrm{g} / \mathrm{mL}$, Sigma) for $20 \mathrm{~min}$ at $37{ }^{\circ} \mathrm{C}$, washed three times and manually triturated in cHBSS supplemented with DNAse. Cells were then plated at 5 or $10 \times 10^{4}$ cells per $35 \mathrm{~mm}$ glass bottom dish (Matek) coated with poly-D-lysine (1 mg/mL, Sigma). Short term cultures without glial cells were cultured for 5-10 days in neurobasal medium (Life Technologies) supplemented with B27 (1X, Life Technologies), $\mathrm{N}_{2}$ (1x, Life Technologies) and L-glutamine ( $2 \mathrm{mM}$, Life Technologies), while long term cultures with glial cells were cultured for 7-21 days in neurobasal medium supplemented with B27 (1X), L-glutamine (2 mM) and $2.5 \%$ Fetal Bovine Serum (Atlanta Biologicals). One third of the medium was changed every 5 days thereafter. Non-glial cell cultures were infected at 5 days in vitro (DIV), while glial cell containing cultures were infected at 5DIV or 19DIV. 
Ex vivo slice culture P3 CD1 coronal brain sections were prepared and cultured as previously published. ${ }^{2}$ Briefly, P3 brains were collected and embedded in cHBSS containing low melting point agarose. After the agarose had solidified the brains were mounted onto the vibratome and $300 \mu \mathrm{m}$ sections were cut. Sections were mounted on falcon culture inserts that had been pre-coated with PDL and laminin and 1.8 $\mathrm{mL}$ of slice culture medium was added to each well. Brain slices were cultured between 24-48 hours after sectioning. See above reference for reagent details and usage. Slices were infected directly after sectioning.

Immunocytochemistry Cultured neurons or brain slices were fixed at 24 or 48 hours post infection with 4\% PFA (EMS) for 10 or 60 minutes respectively then washed three times with PBS (Sigma). After fixation, samples were blocked/permeabilized with blocking solution (1\% BSA, 5\% normal goat serum and $0.1 \%$ Triton X-100 in PBS, Sigma) for 1 hour at room temperature. After blocking, primary antibody was diluted in blocking solution and added for 60 minutes or overnight (at $4{ }^{\circ} \mathrm{C}$ ) respectively. After three washes in PBS, secondary antibody was diluted in blocking solution and added for $60 \mathrm{~min}$ in the dark. Primary antibody concentrations were as follows: chicken anti-GFP (Aves) 1:2,000, rabbit anti-MAP2 (Covance) 1:1,000. Secondary antibodies were conjugated to Alexa 488, 594 or 647 fluorophores (Life Technologies). Hoechst 33258 (Life Technologies) was added at the same time as the secondary antibodies.

A map and full sequence of pAcBac3 is given below. EGFP-39-TAG, tRNA, and aaRS sequences are color-coded with green, red, and blue texts, respectively.

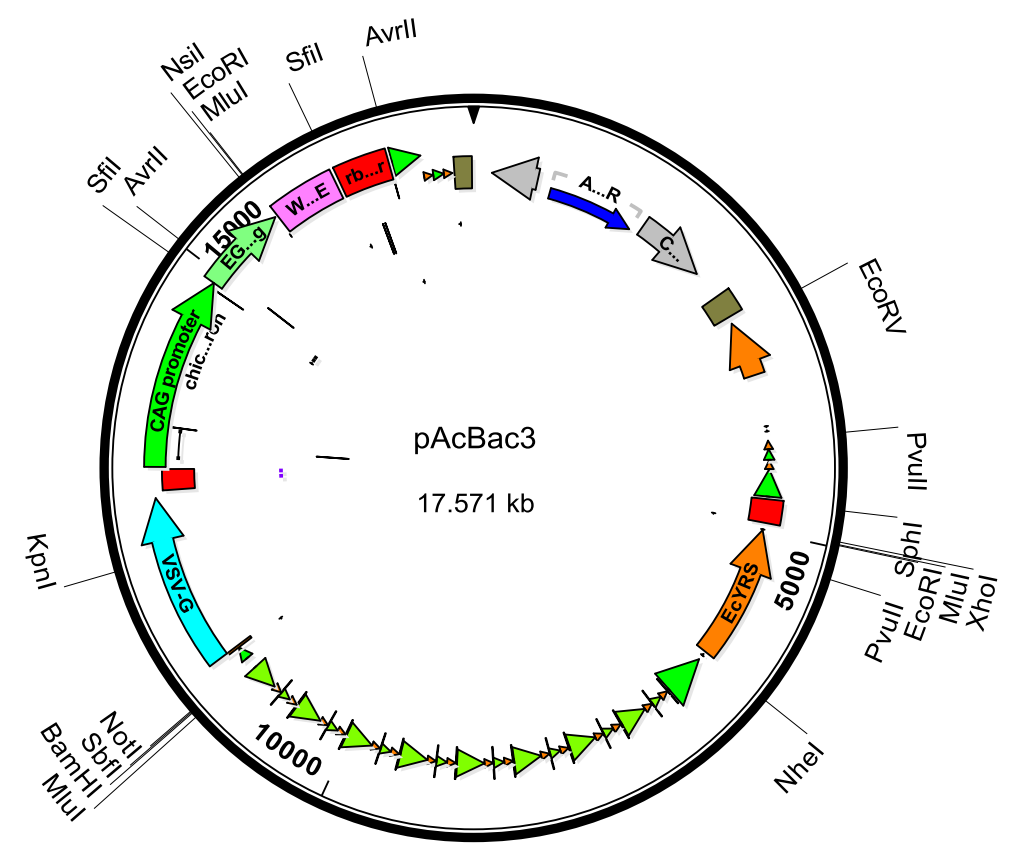

CCTGTTATGTTTTTAATCAAACATCCTGCCAACTCCATGTGACAAACCGTCATCTTCGGCTACTTTTTCTCTGTCACAGAATGAAAA TTTTTCTGTCATCTCTTCGTTATTAATGTTTGTAATTGACTGAATATCAACGCTTATTTGCAGCCTGAATGGCGAATGGGACGCGCC CTGTAGCGGCGCATTAAGCGCGGCGGGTGTGGTGGTTACGCGCAGCGTGACCGCTACACTTGCCAGCGCCCTAGCGCCCGCTCCT TTCGCTTTCTTCCCTTCCTTTCTCGCCACGTTCGCCGGCTTTCCCCGTCAAGCTCTAAATCGGGGGCTCCCTTTAGGGTTCCGATTTA GTGCTTTACGGCACCTCGACCCCAAAAAACTTGATTAGGGTGATGGTTCACGTAGTGGGCCATCGCCCTGATAGACGGTTTTTCGC CCTTTGACGTTGGAGTCCACGTTCTTTAATAGTGGACTCTTGTTCCAAACTGGAACAACACTCAACCCTATCTCGGTCTATTCTTTT GATTTATAAGGGATTTTGCCGATTTCGGCCTATTGGTTAAAAAATGAGCTGATTTAACAAAAATTTAACGCGAATTTTAACAAAAT ATTAACGTTTACAATTTCAGGTGGCACTTTTCGGGGAAATGTGCGCGGAACCCCTATTTGTTTATTTTTCTAAATACATTCAAATAT 
GTATCCGCTCATGAGACAATAACCCTGATAAATGCTTCAATAATATTGAAAAAGGAAGAGTATGAGTATTCAACATTTCCGTGTC GCCCTTATTCCCTTTTTTGCGGCATTTTGCCTTCCTGTTTTTGCTCACCCAGAAACGCTGGTGAAAGTAAAAGATGCTGAAGATCAG TTGGGTGCACGAGTGGGTTACATCGAACTGGATCTCAACAGCGGTAAGATCCTTGAGAGTTTTCGCCCCGAAGAACGTTTTCCAAT GATGAGCACTTTTAAAGTTCTGCTATGTGGCGCGGTATTATCCCGTATTGACGCCGGGCAAGAGCAACTCGGTCGCCGCATACACT ATTCTCAGAATGACTTGGTTGAGTACTCACCAGTCACAGAAAAGCATCTTACGGATGGCATGACAGTAAGAGAATTATGCAGTGC TGCCATAACCATGAGTGATAACACTGCGGCCAACTTACTTCTGACAACGATCGGAGGACCGAAGGAGCTAACCGCTTTTTTGCAC AACATGGGGGATCATGTAACTCGCCTTGATCGTTGGGAACCGGAGCTGAATGAAGCCATACCAAACGACGAGCGTGACACCACG ATGCCTGTAGCAATGGCAACAACGTTGCGCAAACTATTAACTGGCGAACTACTTACTCTAGCTTCCCGGCAACAATTAATAGACT GGATGGAGGCGGATAAAGTTGCAGGACCACTTCTGCGCTCGGCCCTTCCGGCTGGCTGGTTTATTGCTGATAAATCTGGAGCCGG TGAGCGTGGGTCTCGCGGTATCATTGCAGCACTGGGGCCAGATGGTAAGCCCTCCCGTATCGTAGTTATCTACACGACGGGGAGT CAGGCAACTATGGATGAACGAAATAGACAGATCGCTGAGATAGGTGCCTCACTGATTAAGCATTGGTAACTGTCAGACCAAGTTT ACTCATATATACTTTAGATTGATTTAAAACTTCATTTTTAATTTAAAAGGATCTAGGTGAAGATCCTTTTTGATAATCTCATGACCA AAATCCCTTAACGTGAGTTTTCGTTCCACTGAGCGTCAGACCCCGTAGAAAAGATCAAAGGATCTTCTTGAGATCCTTTTTTTCTG CGCGTAATCTGCTGCTTGCAAACAAAAAAACCACCGCTACCAGCGGTGGTTTGTTTGCCGGATCAAGAGCTACCAACTCTTTTTCC GAAGGTAACTGGCTTCAGCAGAGCGCAGATACCAAATACTGTCCTTCTAGTGTAGCCGTAGTTAGGCCACCACTTCAAGAACTCT GTAGCACCGCCTACATACCTCGCTCTGCTAATCCTGTTACCAGTGGCTGCTGCCAGTGGCGATAAGTCGTGTCTTACCGGGTTGGA CTCAAGACGATAGTTACCGGATAAGGCGCAGCGGTCGGGCTGAACGGGGGGTTCGTGCACACAGCCCAGCTTGGAGCGAACGAC CTACACCGAACTGAGATACCTACAGCGTGAGCATTGAGAAAGCGCCACGCTTCCCGAAGGGAGAAAGGCGGACAGGTATCCGGT AAGCGGCAGGGTCGGAACAGGAGAGCGCACGAGGGAGCTTCCAGGGGGAAACGCCTGGTATCTTTATAGTCCTGTCGGGTTTCG CCACCTCTGACTTGAGCGTCGATTTTTGTGATGCTCGTCAGGGGGGCGGAGCCTATGGAAAAACGCCAGCAACGCGGCCTTTTTAC GGTTCCTGGCCTTTTGCTGGCCTTTTGCTCACATGTTCTTTCCTGCGTTATCCCCTGATTCTGTGGATAACCGTATTACCGCCTTTGA GTGAGCTGATACCGCTCGCCGCAGCCGAACGACCGAGCGCAGCGAGTCAGTGAGCGAGGAAGCGGAAGAGCGCCTGATGCGGTA TTTTCTCCTTACGCATCTGTGCGGTATTTCACACCGCAGACCAGCCGCGTAACCTGGCAAAATCGGTTACGGTTGAGTAATAAATG GATGCCCTGCGTAAGCGGGTGTGGGCGGACAATAAAGTCTTAAACTGAACAAAATAGATCTAAACTATGACAATAAAGTCTTAAA CTAGACAGAATAGTTGTAAACTGAAATCAGTCCAGTTATGCTGTGAAAAAGCATACTGGACTTTTGTTATGGCTAAAGCAAACTC TTCATTTTCTGAAGTGCAAATTGCCCGTCGTATTAAAGAGGGGCGTGGCCAAGGGCATGGTAAAGACTATATTCGCGGCGTTGTG ACAATTTACCGAACAACTCCGCGGCCGGGAAGCCGATCTCGGCTTGAACGAATTGTTAGGTGGCGGTACTTGGGTCGATATCAAA GTGCATCACTTCTTCCCGTATGCCCAACTTTGTATAGAGAGCCACTGCGGGATCGTCACCGTAATCTGCTTGCACGTAGATCACAT AAGCACCAAGCGCGTTGGCCTCATGCTTGAGGAGATTGATGAGCGCGGTGGCAATGCCCTGCCTCCGGTGCTCGCCGGAGACTGC GAGATCATAGATATAGATCTCACTACGCGGCTGCTCAAACCTGGGCAGAACGTAAGCCGCGAGAGCGCCAACAACCGCTTCTTGG TCGAAGGCAGCAAGCGCGATGAATGTCTTACTACGGAGCAAGTTCCCGAGGTAATCGGAGTCCGGCTGATGTTGGGAGTAGGTGG CTACGTCTCCGAACTCACGACCGAAAAGATCAAGAGCAGCCCGCATGGATTTGACTTGGTCAGGGCCGAGCCTACATGTGCGAAT GATGCCCATACTTGAGCCACCTAACTTTGTTTTAGGGCGACTGCCCTGCTGCGTAACATCGTTGCTGCTGCGTAACATCGTTGCTG CTCCATAACATCAAACATCGACCCACGGCGTAACGCGCTTGCTGCTTGGATGCCCGAGGCATAGACTGTACAAAAAAACAGTCAT AACAAGCCATGAAAACCGCCACTGCGCCGTTACCACCGCTGCGTTCGGTCAAGGTTCTGGACCAGTTGCGTGAGCGCATACGCTA CTTGCATTACAGTTTACGAACCGAACAGGCTTATGTCAACTGGGTTCGTGCCTTCATCCGTTTCCACGGTGTGCGTCACCCGGCAA CCTTGGGCAGCAGCGAAGTCGAGGCATTTCTGTCCTGGCTGGCGAACGAGCGCAAGGTTTCGGTCTCCACGCATCGTCAGGCATT GGCGGCCTTGCTGTTCTTCTACGGCAAGGTGCTGTGCACGGATCTGCCCTGGCTTCAGGAGATCGGTAGACCTCGGCCGTCGCGGC GCTTGCCGGTGGTGCTGACCCCGGATGAAGTGGTTCGCATCCTCGGTTTTCTGGAAGGCGAGCATCGTTTGTTCGCCCAGGACTCT AGCTATAGTTCTAGTGGTTGGCTACGTACCCGTAGTGGCTATGGCAGGGCTTGCGCTTAATGCGCCGCTACAGGGCGCGTGGGGA TACCCCCTAGAGCCCCAGCTGAAAAAATGGAGGGGGACGGATTCGAACCGCCGAACCCAAAGGGAGCGGATTTAGAGTCCGCCG CGTTTAGCCACTTCGCTACCCCTCCGGTGTCTCTATCACTGATAGGGAACTTATAAGTCTCTATCACTGATAGGGATTTCACGTTTA TGGTGATTTCCCAGAACACATAGCGACATGCAAATATTAAAAAATGGTGGGGGAAGGATTCGAACCTTCGAAGTCTGTGACGGCA GATTTAGAGTCTGCTCCCTTTGGCCGCTCGGGAACCCCACCGGTGTTTCGTCCTTTCCACAAGATATATAAAGCCAAGAAATCGAA ATACTTTCAAGTTACGGTAAGCATATGATAGTCCATTTTAAAACATAATTTTAAAACTGCAAACTACCCAAGAAATTATTACTTTC TACGTCACGTATTTTGTACTAATATCTTTGTGTTTACAGTCAAATTAATTCTAATTATCTCTCTAACAGCCTTGTATCGTATATGCA AATATGAAGGAATCATGGGAAATAGGCCCTCTTCCTGCCCGAGATCTGGTTCTTTCCGCCTCAGAAGCCATAGAGCCCACCGCAT CCCCAGCATGCCTGCTATTGTCTTCCCAATCCTCCCCCTTGCTGTCCTGCCCCACCCCACCCCCCAGAATAGAATGACACCTACTCA GACAATGCGATGCAATTTCCTCATTTTATTAGGAAAGGACAGTGGGAGTGGCACCTTCCAGGGTCAAGGAAGGCACGGGGGAGG GGCAAACAACAGATGGCTGGCAACTAGAAGGCACAGTCGAGGCTGATCAGCGGGTTTAAACGGGCCCTCTAGACTCGAGTTAAA GTCGACTTAACGCGTTGAATTCTTAAACGGGCCCTTTCCAGCAAATCAGACAGTAATTCTTTTTACCGCGACGCAGTAAGGTAAAA CGACCAAACAGACGATCTTCTTCTTTAAAGAAGTATTCAGGATCGGACTGTTTTTCACCGTTAATGGTGATGGCATTGGAGGCGAT AGTTTTACGTGCCTGACCACGGGAAGGTTGCAGTTCAGAATCGACCAGTGCCTGCATCAGGTCTGCGCCCTTTTCCATCTCAACCA TCGGTACGCCGTCCTGCGCCAGCTGTTCGAAGTCCGCTTCACTCAGCGCACTCAAAGAACCGCTGAACAGGCATTCGGTAATACG TTTTGCCGCCTGTAAACCTTCTTCACCGTGAACCAGACGAGTCACCTGCTCCGCCAGTACATACTGGGCGCGCGGTGCTTTACCGC TGTTTTTATCTTCTTCTTCCAGGGCGTTGATCTCTTCAATGCTCATAAAGGTGAAGAACTTCAGGAAGCGGTAAACGTCGGCATCC GCAGTGTTGATCCAGAACTGGTAGAATTTGTACGGGCTGGTTTTCTTCGGGTCCAACCAGACTGCGCCGCCTTCAGTTTTACCAAA TTTGGTGCCATCTGCTTTAGTGATCAGCGGAACGGTCAGGCCAAACACCTGATTCTGATGCAGACGACGGGTCAGGTCGATACCA GAAGTGATGTTACCCCATTGGTCAGAACCACCAATTTGCAGCACCACACCGTACTGTTTGTTAGCACAGGCCATACCATAACCCTG GAGCAGGTTGTAGGAAAACTCAGTGAACGAAATCCCCTGACCTTCACGGTTGAGACGCTGCTTAACCGCTTCTTTGTTGATCATCT GGTTAACGGAGAAGTGTTTGCCAATATCGCGCAGGAAGGTCAGCACATTCATATTGCCGAACCAGTCATAATTATTGGCCGCGAT 
AGCAGAGTTTTCTCCACAGTCGAAATCGAGGAACGGGGCAACCTGCTTACGGATTTTGTCCACCCACTCCTGAACAGTTTCTTCGG TGTTCAGCTTACGCTCGGCAGCTTTGAAGCTCGGGTCGCCAATCAGACCCGTCGCGCCGCCTACCAGCGCAACCGGCTTGTGGCCC GCCTGCTGGAAGCGTTTCAGGCATAACAATGGAACAAGATGCCCCAAATGCAAGCTGTCAGCGGTAGGATCGAAGCCACAAATG AGTGCGATCGGGCCTTGCGCCAGTCGCTCTGCTAACGCTTCCTCGTCCGTCACCTGGGCTACCAGCCCCCGCTCTTGCAATTGTTT AATCAAGTTACTGCTTGCCATGGTGGCGCTAGCCAGCTTGGGTCTCCCTATAGTGAGTCGTATTAATTTCGATAAGCCAGTAAGCA GTGGGTTCTCTAGTTAGCCAGAGAGCTCTAGACCAAGTGACGATCACAGCGATCCACAAACAAGAACCGCGACCCAAATCCCGG CTGCGACGGAACTAGCTGTGCCACACCCGGCGCGTCCTTATATAATCATCGGCGTTCACCGCCCCACGGAGATCCCTCCGCAGAA TCGCCGAGAAGGGACTACTTTTCCTCGCCTGTTCCGCTCTCTGGAAAGAAAACCAGTGCCCTAGAGTCACCCAAGTCCCGTCCTAA AATGTCCTTCTGCTGATACTGGGGTTCTAAGGCCGAGTCTTATGAGCAGCGGGCCGCTGTCCTGAGCGTCCGGGCGGAAGGATCA GGACGCTCGCTGCGCCCTTCGTCTGACGTGGCAGCGCTCGCCGTGAGGAGGGGGGCGCCCGCGGGAGGCGCCAAAACCCGGCGC GGAGGCCTTCGAACGGCCACTAGCAAAAAATGGAGGGGGACGGATTCGAACCGCCGAACCCAAAGGGAGCGGATTTAGAGTCCG CCGCGTTTAGCCACTTCGCTACCCCTCCGGTGTCTCTATCACTGATAGGGAACTTATAAGTCTCTATCACTGATAGGGATTTCACGT TTATGGTGATTTCCCAGAACACATAGCGACATGCAAATATTAAAAAATGGTGGGGGAAGGATTCGAACCTTCGAAGTCTGTGACG GCAGATTTAGAGTCTGCTCCCTTTGGCCGCTCGGGAACCCCACCGGTGTTTCGTCCTTTCCACAAGATATATAAAGCCAAGAAATC GAAATACTTTCAAGTTACGGTAAGCATATGATAGTCCATTTTAAAACATAATTTTAAAACTGCAAACTACCCAAGAAATTATTACT TTCTACGTCACGTATTTTGTACTAATATCTTTGTGTTTACAGTCAAATTAATTCTAATTATCTCTCTAACAGCCTTGTATCGTATATG CAAATATGAAGGAATCATGGGAAATAGGCCCTCTTCCTGCCCGACCTAGCAAAAAATGGAGGGGGACGGATTCGAACCGCCGAA CCCAAAGGGAGCGGATTTAGAGTCCGCCGCGTTTAGCCACTTCGCTACCCCTCCGGTGTCTCTATCACTGATAGGGAACTTATAAG TCTCTATCACTGATAGGGATTTCACGTTTATGGTGATTTCCCAGAACACATAGCGACATGCAAATATTAAAAAATGGTGGGGGAA GGATTCGAACCTTCGAAGTCTGTGACGGCAGATTTAGAGTCTGCTCCCTTTGGCCGCTCGGGAACCCCACCGGTGTTTCGTCCTTT CCACAAGATATATAAAGCCAAGAAATCGAAATACTTTCAAGTTACGGTAAGCATATGATAGTCCATTTTAAAACATAATTTTAAA ACTGCAAACTACCCAAGAAATTATTACTTTCTACGTCACGTATTTTGTACTAATATCTTTGTGTTTACAGTCAAATTAATTCTAATT ATCTCTCTAACAGCCTTGTATCGTATATGCAAATATGAAGGAATCATGGGAAATAGGCCCTCTTCCTGCCCGACCTAGCAAAAAAT GGAGGGGGACGGATTCGAACCGCCGAACCCAAAGGGAGCGGATTTAGAGTCCGCCGCGTTTAGCCACTTCGCTACCCCTCCGGTG TCTCTATCACTGATAGGGAACTTATAAGTCTCTATCACTGATAGGGATTTCACGTTTATGGTGATTTCCCAGAACACATAGCGACA TGCAAATATTAAAAAATGGTGGGGGAAGGATTCGAACCTTCGAAGTCTGTGACGGCAGATTTAGAGTCTGCTCCCTTTGGCCGCT CGGGAACCCCACCGGTGTTTCGTCCTTTCCACAAGATATATAAAGCCAAGAAATCGAAATACTTTCAAGTTACGGTAAGCATATG ATAGTCCATTTTAAAACATAATTTTAAAACTGCAAACTACCCAAGAAATTATTACTTTCTACGTCACGTATTTTGTACTAATATCTT TGTGTTTACAGTCAAATTAATTCTAATTATCTCTCTAACAGCCTTGTATCGTATATGCAAATATGAAGGAATCATGGGAAATAGGC ССТCTTCCTGCCCGACCTAGCAAAAAATGGAGGGGGACGGATTCGAACCGCCGAACCCAAAGGGAGCGGATTTAGAGTCCGCCG CGTTTAGCCACTTCGCTACCCCTCCGGTGTCTCTATCACTGATAGGGAACTTATAAGTCTCTATCACTGATAGGGATTTCACGTTTA TGGTGATTTCCCAGAACACATAGCGACATGCAAATATTAAAAAATGGTGGGGGAAGGATTCGAACCTTCGAAGTCTGTGACGGCA GATTTAGAGTCTGCTCCCTTTGGCCGCTCGGGAACCCCACCGGTGTTTCGTCCTTTCCACAAGATATATAAAGCCAAGAAATCGAA ATACTTTCAAGTTACGGTAAGCATATGATAGTCCATTTTAAAACATAATTTTAAAACTGCAAACTACCCAAGAAATTATTACTTTC TACGTCACGTATTTTGTACTAATATCTTTGTGTTTACAGTCAAATTAATTCTAATTATCTCTCTAACAGCCTTGTATCGTATATGCA AATATGAAGGAATCATGGGAAATAGGCCCTCTTCCTGCCCGACCTAGCAAAAAATGGAGGGGGACGGATTCGAACCGCCGAACC CAAAGGGAGCGGATTTAGAGTCCGCCGCGTTTAGCCACTTCGCTACCCCTCCGGTGTCTCTATCACTGATAGGGAACTTATAAGTC TCTATCACTGATAGGGATTTCACGTTTATGGTGATTTCCCAGAACACATAGCGACATGCAAATATTAAAAAATGGTGGGGGAAGG ATTCGAACCTTCGAAGTCTGTGACGGCAGATTTAGAGTCTGCTCCCTTTGGCCGCTCGGGAACCCCACCGGTGTTTCGTCCTTTCC ACAAGATATATAAAGCCAAGAAATCGAAATACTTTCAAGTTACGGTAAGCATATGATAGTCCATTTTAAAACATAATTTTAAAAC TGCAAACTACCCAAGAAATTATTACTTTCTACGTCACGTATTTTGTACTAATATCTTTGTGTTTACAGTCAAATTAATTCTAATTAT CTCTCTAACAGCCTTGTATCGTATATGCAAATATGAAGGAATCATGGGAAATAGGCCCTCTTCCTGCCCGACCTAGCAAAAAATG GAGGGGGACGGATTCGAACCGCCGAACCCAAAGGGAGCGGATTTAGAGTCCGCCGCGTTTAGCCACTTCGCTACCCCTCCGGTGT CTCTATCACTGATAGGGAACTTATAAGTCTCTATCACTGATAGGGATTTCACGTTTATGGTGATTTCCCAGAACACATAGCGACAT GCAAATATTAAAAAATGGTGGGGGAAGGATTCGAACCTTCGAAGTCTGTGACGGCAGATTTAGAGTCTGCTCCCTTTGGCCGCTC GGGAACCCCACCGGTGTTTCGTCCTTTCCACAAGATATATAAAGCCAAGAAATCGAAATACTTTCAAGTTACGGTAAGCATATGA TAGTCCATTTTAAAACATAATTTTAAAACTGCAAACTACCCAAGAAATTATTACTTTCTACGTCACGTATTTTGTACTAATATCTTT GTGTTTACAGTCAAATTAATTCTAATTATCTCTCTAACAGCCTTGTATCGTATATGCAAATATGAAGGAATCATGGGAAATAGGCC CTCTTCCTGCCCGACCTAGCAAAAAATGGAGGGGGACGGATTCGAACCGCCGAACCCAAAGGGAGCGGATTTAGAGTCCGCCGC GTTTAGCCACTTCGCTACCCCTCCGGTGTCTCTATCACTGATAGGGAACTTATAAGTCTCTATCACTGATAGGGATTTCACGTTTAT GGTGATTTCCCAGAACACATAGCGACATGCAAATATTAAAAAATGGTGGGGGAAGGATTCGAACCTTCGAAGTCTGTGACGGCA GATTTAGAGTCTGCTCCCTTTGGCCGCTCGGGAACCCCACCGGTGTTTCGTCCTTTCCACAAGATATATAAAGCCAAGAAATCGAA ATACTTTCAAGTTACGGTAAGCATATGATAGTCCATTTTAAAACATAATTTTAAAACTGCAAACTACCCAAGAAATTATTACTTTC TACGTCACGTATTTTGTACTAATATCTTTGTGTTTACAGTCAAATTAATTCTAATTATCTCTCTAACAGCCTTGTATCGTATATGCA AATATGAAGGAATCATGGGAAATAGGCCCTCTTCCTGCCCGACCTAGCAAAAAATGGAGGGGGACGGATTCGAACCGCCGAACC CAAAGGGAGCGGATTTAGAGTCCGCCGCGTTTAGCCACTTCGCTACCCCTCCGGTGTCTCTATCACTGATAGGGAACTTATAAGTC TCTATCACTGATAGGGATTTCACGTTTATGGTGATTTCCCAGAACACATAGCGACATGCAAATATTAAAAAATGGTGGGGGAAGG ATTCGAACCTTCGAAGTCTGTGACGGCAGATTTAGAGTCTGCTCCCTTTGGCCGCTCGGGAACCCCACCGGTGTTTCGTCCTTTCC ACAAGATATATAAAGCCAAGAAATCGAAATACTTTCAAGTTACGGTAAGCATATGATAGTCCATTTTAAAACATAATTTTAAAAC TGCAAACTACCCAAGAAATTATTACTTTCTACGTCACGTATTTTGTACTAATATCTTTGTGTTTACAGTCAAATTAATTCTAATTAT CTCTCTAACAGCCTTGTATCGTATATGCAAATATGAAGGAATCATGGGAAATAGGCCCTCTTCCTGCCCGACCTAGTCAATAATCA 
ATGTCAACGCGTATATCTGGCCCGTACATCGCGAAGCAGCGCAAAACGGATCCTGCAGGTATTTGCGGCCGCGGTCCGTATACTC CGGAATATTAATAGATCATGGAGATAATTAAAATGATAACCATCTCGCAAATAAATAAGTATTTTACTGTTTTCGTAACAGTTTTG TAATAAAAAAACCTATAAATATTCCGGATTATTCATACCGTCCCACCATCGGGCGCGAACTCCTAAAAAACCGCCACCATGAAGT GCCTTTTGTACTTAGCCTTTTTATTCATTGGGGTGAATTGCAAGTTCACCATAGTTTTTCCACACAACCAAAAAGGAAACTGGAAA AATGTTCCTTCTAATTACCATTATTGCCCGTCAAGCTCAGATTTAAATTGGCATAATGACTTAATAGGCACAGCCTTACAAGTCAA AATGCCCAAGAGTCACAAGGCTATTCAAGCAGACGGTTGGATGTGTCATGCTTCCAAATGGGTCACTACTTGTGATTTCCGCTGGT ATGGACCGAAGTATATAACACATTCCATCCGATCCTTCACTCCATCTGTAGAACAATGCAAGGAAAGCATTGAACAAACGAAACA AGGAACTTGGCTGAATCCAGGCTTCCCTCCTCAAAGTTGTGGATATGCAACTGTGACGGATGCCGAAGCAGTGATTGTCCAGGTG ACTCCTCACCATGTGCTGGTTGATGAATACACAGGAGAATGGGTTGATTCACAGTTCATCAACGGAAAATGCAGCAATTACATAT GCCCCACTGTCCATAACTCTACAACCTGGCATTCTGACTATAAGGTCAAAGGGCTATGTGATTCTAACCTCATTTCCATGGACATC ACCTTCTTCTCAGAGGACGGAGAGCTATCATCCCTGGGAAAGGAGGGCACAGGGTTCAGAAGTAACTACTTTGCTTATGAAACTG GAGGCAAGGCCTGCAAAATGCAATACTGCAAGCATTGGGGAGTCAGACTCCCATCAGGTGTCTGGTTCGAGATGGCTGATAAGG ATCTCTTTGCTGCAGCCAGATTCCCTGAATGCCCAGAAGGGTCAAGTATCTCTGCTCCATCTCAGACCTCAGTGGATGTAAGTCTA ATTCAGGACGTTGAGAGGATCTTGGATTATTCCCTCTGCCAAGAAACCTGGAGCAAAATCAGAGCGGGTCTTCCAATCTCTCCAGT GGATCTCAGCTATCTTGCTCCTAAAAACCCAGGAACCGGTCCTGCTTTCACCATAATCAATGGTACCCTAAAATACTTTGAGACCA GATACATCAGAGTCGATATTGCTGCTCCAATCCTCTCAAGAATGGTCGGAATGATCAGTGGAACTACCACAGAAAGGGAACTGTG GGATGACTGGGCACCATATGAAGACGTGGAAATTGGACCCAATGGAGTTCTGAGGACCAGTTCAGGATATAAGTTTCCTTTATAC ATGATTGGACATGGTATGTTGGACTCCGATCTTCATCTTAGCTCAAAGGCTCAGGTGTTCGAACATCCTCACATTCAAGACGCTGC TTCGCAACTTCCTGATGATGAGAGTTTATTTTTTGGTGATACTGGGCTATCCAAAAATCCAATCGAGCTTGTAGAAGGTTGGTTCA GTAGTTGGAAAAGCTCTATTGCCTCTTTTTTCTTTATCATAGGGTTAATCATTGGACTATTCTTGGTTCTCCGAGTTGGTATCCATCT TTGCATTAAATTAAAGCACACCAAGAAAAGACAGATTTATACAGACATAGAGATGAACCGACTTGGAAAGTGATAAGTCGAGAA GTACTAGAGGATCATAATCAGCCATACCACATTTGTAGAGGTTTTACTTGCTTTAAAAAACCTCCCACACCTCCCCCTGAACCTGA AACATAAAATGAATGCAATTGTTGTTGTTAACTTGTTTATTGCAGCTTATAATGGTTACAAATAAAGCAATAGCATCACAAATTTC ACAAATAAAGCATTTTTTTCACTGCATTCTAGTTGTGGTTTGTCCAAACTCATCAATGTATCTTATCATGTCTGGATCTGATCACTG CTTGAGCCTAGTTATTAATAGTAATCAATTACGGGGTCATTAGTTCATAGCCCATATATGGAGTTCCGCGTTACATAACTTACGGT AAATGGCCCGCCTGGCTGACCGCCCAACGACCCCCGCCCATTGACGTCAATAATGACGTATGTTCCCATAGTAACGCCAATAGGG ACTTTCCATTGACGTCAATGGGTGGAGTATTTACGGTAAACTGCCCACTTGGCAGTACATCAAGTGTATCATATGCCAAGTACGCC CCCTATTGACGTCAATGACGGTAAATGGCCCGCCTGGCATTATGCCCAGTACATGACCTTATGGGACTTTCCTACTTGGCAGTACA TCTACGTATTAGTCATCGCTATTACCATGGTCGAGGTGAGCCCCACGTTCTGCTTCACTCTCCCCATCTCCCCCCCCTCCCCACCCC CAATTTTGTATTTATTTATTTTTTAATTATTTTGTGCAGCGATGGGGGCGGGGGGGGGGGGGGCGCGCGCCAGGCGGGGCGGGGC GGGGCGAGGGGCGGGGCGGGGCGAGGCGGAGAGGTGCGGCGGCAGCCAATCAGAGCGGCGCGCTCCGAAAGTTTCCTTTTATGG CGAGGCGGCGGCGGCGGCGGCCCTATAAAAAGCGAAGCGCGCGGCGGGCGGGAGTCGCTGCGTTGCCTTCGCCCCGTGCCCCGC TCCGCGCCGCCTCGCGCCGCCCGCCCCGGCTCTGACTGACCGCGTTACTCCCACAGGTGAGCGGGCGGGACGGCCCTTCTCCTCCG GGCTGTAATTAGCGCTTGGTTTAATGACGGCTCGTTTCTTTTCTGTGGCTGCGTGAAAGCCTTAAAGGGCTCCGGGAGGGCCCTTT GTGCGGGGGGGAGCGGCTCGGGGGGTGCGTGCGTGTGTGTGTGCGTGGGGAGCGCCGCGTGCGGCCCGCGCTGCCCGGCGGCTG TGAGCGCTGCGGGCGCGGCGCGGGGCTTTGTGCGCTCCGCGTGTGCGCGAGGGGAGCGCGGCCGGGGGCGGTGCCCCGCGGTGC GGGGGGGCTGCGAGGGGAACAAAGGCTGCGTGCGGGGTGTGTGCGTGGGGGGGTGAGCAGGGGGTGTGGGCGCGGCGGTCGGG CTGTAACCCCCCCCTGCACCCCCCTCCCCGAGTTGCTGAGCACGGCCCGGCTTCGGGTGCGGGGCTCCGTGCGGGGCGTGGCGCG GGGCTCGCCGTGCCGGGCGGGGGGTGGCGGCAGGTGGGGGTGCCGGGCGGGGCGGGGCCGCCTCGGGCCGGGGAGGGCTCGGG GGAGGGGCGCGGCGGCCCCGGAGCGCCGGCGGCTGTCGAGGCGCGGCGAGCCGCAGCCATTGCCTTTTATGGTAATCGTGCGAG AGGGCGCAGGGACTTCCTTTGTCCCAAATCTGGCGGAGCCGAAATCTGGGAGGCGCCGCCGCACCCCCTCTAGCGGGCGCGGGCG AAGCGGTGCGGCGCCGGCAGGAAGGAAATGGGCGGGGAGGGCCTTCGTGCGTCGCCGCGCCGCCGTCCCCTTCTCCATCTCCAGC CTCGGGGCTGCCGCAGGGGGACGGCTGCCTTCGGGGGGGACGGGGCAGGGCGGGGTTCGGCTTCTGGCGTGTGACCGGCGGCTC TAGAGCCTCTGCTAACCATGTTCATGCCTTCTTCTTTTTCCTACAGCTCCTGGGCAACGTGCTGGTTATTGTGCTGTCTCATCATTTT GGCAAAGAATTGGCCAAGGAGGCCACCATGGTGAGCAAGGGCGAGGAGCTGTTCACCGGGGTGGTGCCCATCCTGGTCGAGCTG GACGGCGACGTAAACGGCCACAAGTTCAGCGTGTCCGGCGAGGGCGAGGGCGATGCCACCTAGGGCAAGCTGACCCTGAAGTTC ATCTGCACCACCGGCAAGCTGCCCGTGCCCTGGCCCACCCTCGTGACCACCCTGACCTACGGCGTGCAGTGCTTCAGCCGCTACCC CGACCACATGAAGCAGCACGACTTCTTCAAGTCCGCCATGCCCGAAGGCTACGTCCAGGAGCGCACCATCTTCTTCAAGGACGAC GGCAACTACAAGACCCGCGCCGAGGTGAAGTTCGAGGGCGACACCCTGGTGAACCGCATCGAGCTGAAGGGCATCGACTTCAAG GAGGACGGCAACATCCTGGGGCACAAGCTGGAGTACAACTACAACAGCCACAACGTCTATATCATGGCCGACAAGCAGAAGAAC GGCATCAAGGTGAACTTCAAGATCCGCCACAACATCGAGGACGGCAGCGTGCAGCTCGCCGACCACTACCAGCAGAACACCCCC ATCGGCGACGGCCCCGTGCTGCTGCCCGACAACCACTACCTGAGCACCCAGTCCGCCCTGAGCAAAGACCCCAACGAGAAGCGC GATCACATGGTCCTGCTGGAGTTCGTGACCGCCGCCGGGATCACTCTCGGCATGGACGAGCTGTACAAGGGGCCCTTCGAACAAA AACTCATCTCAGAAGAGGATCTGAATATGCATACCGGTCATCATCACCATCACCATTGAGAATTCAACGCGTTAAGTCGACAATC AACCTCTGGATTACAAAATTTGTGAAAGATTGACTGGTATTCTTAACTATGTTGCTCCTTTTACGCTATGTGGATACGCTGCTTTAA TGCCTTTGTATCATGCTATTGCTTCCCGTATGGCTTTCATTTTCTCCTCCTTGTATAAATCCTGGTTGCTGTCTCTTTATGAGGAGTT GTGGCCCGTTGTCAGGCAACGTGGCGTGGTGTGCACTGTGTTTGCTGACGCAACCCCCACTGGTTGGGGCATTGCCACCACCTGTC AGCTCCTTTCCGGGACTTTCGCTTTCCCCCTCCCTATTGCCACGGCGGAACTCATCGCCGCCTGCCTTGCCCGCTGCTGGACAGGG GCTCGGCTGTTGGGCACTGACAATTCCGTGGTGTTGTCGGGGAAATCATCGTCCTTTCCTTGGCTGCTCGCCTGTGTTGCCACCTGG ATTCTGCGCGGGACGTCCTTCTGCTACGTCCCTTCGGCCCTCAATCCAGCGGACCTTCCTTCCCGCGGCCTGCTGCCGGCTCTGCG GCCTCTTCCGCGTCTTCGCCTTCGCCCTCAGACGAGTCGGATCTCCCTTTGGGCCGCCTCCCCGCGGCCTGGCCGGCCGCCAGCAC 
AGTGGTCGATCGACCAATGCCCTGGCTCACAAATACCACTGAGATCTTTTTCCCTCTGCCAAAAATTATGGGGACATCATGAAGCC CCTTGAGCATCTGACTTCTGGCTAATAAAGGAAATTTATTTTCATTGCAATAGTGTGTTGGAATTTTTTGTGTCTCTCACTCGGAAG GACATATGGGAGGGCAAATCATTTAAAACATCAGAATGAGTATTTGGTTTAGAGTTTGGCAACATATGCCCATATGCTGGCTGCC ATGAACAAAGGTTGGCTATAAAGAGGTCATCAGTATATGAAACAGCCCCCTGCTGTCCATTCCTTATTCCATAGAAAAGCCTTGA CTTGAGGTTAGATTTTTTTTATATTTTGTTTTGTGTTATTTTTTTCTTTAACATCCCTAAAATTTTCCTTACATGTTTTACTAGCCAGA TTTTTCCTCCTCTCCTGACTACTCCCAGTCATAGCTGTCCCTCTTCTCTTATGGAGATCCCTCGACCTGCCCTAGGTCGGGCAGGAA GAGGGCCTATTTCCCATGATTCCTTCATATTTGCATATACGATACAAGGCTGTTAGAGAGATAATTAGAATTAATTTGACTGTAAA CACAAAGATATTAGTACAAAATACGTGACGTAGAAAGTAATAATTTCTTGGGTAGTTTGCAGTTTTAAAATTATGTTTTAAAATGG ACTATCATATGCTTACCGTAACTTGAAAGTATTTCGATTTCTTGGCTTTATATATCTTGTGGAAAGGACGAAACACCGGTGGGGTT CCCGAGCGGCCAAAGGGAGCAGACTCTAAATCTGCCGTCACAGACTTCGAAGGTTCGAATCCTTCCCCCACCATTTTTTAATATTT GCATGTCGCTATGTGTTCTGGGAAATCACCATAAACGTGAAATCCCTATCAGTGATAGAGACTTATAAGTTCCCTATCAGTGATAG AGACACCGGAGGGGTAGCGAAGTGGCTAAACGCGGCGGACTCTAAATCCGCTCCCTTTGGGTTCGGCGGTTCGAATCCGTCCCCC TCCATTTTTTGCTAGGAGATCCGAACCAGATAAGTGAAATCTAGTTCCAAACTATTTTGTCATTTTTAATTTTCGTATTAGCTTACG ACGCTACACCCAGTTCCCATCTATTTTGTCACTCTTCCCTAAATAATCCTTAAAAACTCCATTTCCACCCCTCCCAGTTCCCAACTA TTTTGTCCGCCCACAGCGGGGCATTTTTCTT

\section{References:}

1. A. Chatterjee, H. Xiao, M. Bollong, H. W. Ai and P. G. Schultz, Proceedings of the National Academy of Sciences of the United States of America, 2013, 110, 11803-11808.

2. F. Polleux and A. Ghosh, Science's STKE : signal transduction knowledge environment, 2002, p19 